\title{
COMPUTATIONAL INVESTIGATION OF THE EFFECT OF THE ASPECT RATIO ON SECONDARY CURRENTS IN OPEN CHANNELS
}

\author{
MONSIF SHINNEEB ${ }^{1}$, GHASSAN NASIF ${ }^{1,2}$ \& RAM BALACHANDAR ${ }^{2}$ \\ ${ }^{1}$ Department of Mechanical Engineering, Higher Colleges of Technology, UAE \\ ${ }^{2}$ Department of Mechanical Engineering, University of Windsor, Canada
}

\begin{abstract}
This study investigates numerically the effect of the aspect ratio (AR) on the velocity field characteristics of the turbulent flow of a straight open channel flow. The AR is defined as the ratio of the width of the channel in a plane normal to the flow direction, to the flow depth. In this study, two aspect ratio cases are investigated; a narrow case of $A R=1$ and a wide case of $A R=12$. The transient three-dimensional Navier-Stokes equations were numerically solved using a finite-volume approach with detached-eddy simulation (DES) turbulence model. The free surface was simulated using flatwave model linked with the volume of fluid method. The objective of this study is to enhance our understanding of the effect of the AR on the formation of secondary currents in a channel flow. The results revealed the formation of a pair of counter-rotating recirculation zones near the bottom corners of the channel, whose axes are aligned with the main flow direction. The AR appears to significantly influence the size and strength of the recirculation zone that resides near the sidewalls. The distribution of the turbulent kinetic energy of the flow appears quantitatively similar for both the AR cases; however, the magnitude appears to increase with decreasing the AR.
\end{abstract}

Keywords: secondary currents, turbulence, DES model.

\section{INTRODUCTION}

Turbulent flow is a very complex phenomenon it is one of the most challenging topics in fluid mechanics. Moreover, the presence of solid walls bounding the flow causes the formation of boundary layer, which makes the flow three-dimensional (3D), and thus result in a more intricate flow field due to the wall effect. One of the interesting phenomenon in turbulent flow is the formation of secondary currents. The strength of the secondary currents in the flow field depends on different parameters such as the aspect ratio (AR), the flow depth, wall roughness, the distance from the solid boundary, Reynolds number, etc. This phenomenon is usually observed in channel and duct flows. Therefore, understanding the mechanism of these currents is crucial since they affect the characteristics of turbulence and mean flow.

Open-channel flow is a suitable case to understand the characteristics of the secondary currents. Generally, there are two standard types of secondary currents classified in openchannels [1]. The secondary currents that occur in a straight open channel flow due to turbulence phenomenon is known as Prandtl's second kind, which is the focus of this study. It is reported that the second type of secondary currents occurs due to turbulence anisotropy between velocity components [2]. Nezu and Rodi [3] carried out an experimental study on secondary currents in smooth open-channel flows using a laser-Doppler anemometer (LDA). It was concluded that the secondary current patterns are different from those of duct flows due to the presence of the free-surface. Nezu et al. [4] investigated the formation of secondary currents in a smooth rectangular open channel flow by varying the aspect ratio. It was reported that the secondary currents develop along the corner bisector $\left(45^{\circ}\right.$-line) regardless of the aspect ratio (AR), and it produces a pair of recirculation zones. One recirculation zone resides above the corner bisector-line which is called the "side-vortex," and the other one 
resides under the bisector-line which is called the "bottom-vortex." The side- and bottomvortices are symmetrical with each other with respect to the bisector for aspect ratio $\mathrm{AR}=2.0$ [4]. The size of the side-vortex is completely restricted by the sidewall, and therefore it is not often affected by the AR of the open-channel. On the other hand, the bottom-vortex is greatly influenced by the AR. As AR increases, the size of the bottomvortex expands and then tends to reach a constant size at larger aspect ratios. Nezu and Nakagawa [5] provided an explanation of the dependence of the secondary currents on the channel AR. In narrow channels (AR $<5)$, the sidewalls and bed cause an increased anisotropy between fluctuating velocity components, which result in strong secondary currents over the entire channel cross-section. For AR $>10$, it is argued that the sidewall effects die out and the flow becomes two-dimensional in the central/core region of the channel. Tominaga et al. [6] carried out an experimental study using a hot-film anemometer (HWA) to investigate the secondary currents in rectangular and trapezoidal cross-section open-channel flows. It was concluded that the secondary currents are generated as a result of the anisotropy of turbulence caused by the boundary conditions, and that the secondary currents affect the mean streamwise flow, producing three-dimensional flow.

Even though the secondary currents in turbulent flow have been extensively investigated by numerous researchers, there has still been considerable controversy between researchers about the mechanism of the flow and the influence of the geometry parameters such as the aspect ratio on the formation of the secondary currents. This may be attributed to the threedimensional nature of the turbulent flow and the inherent limitations and high cost of conventional experimental methods, which limits their effectiveness in such studies compared to numerical approaches. The availability of enhanced computational tools makes the investigation of the secondary currents in the flow field more efficient. Thus, the objective of this study is to enhance our understanding of the effect of the AR on the formation of secondary currents in a smooth open-channel flow using a numerical approach. This is achieved by contrasting the mean velocity field and turbulence characteristics at low and high $A R$. Two aspect ratio cases are investigated in this study; a narrow case of $A R=1$ and a wide case of $\mathrm{AR}=12$. A three-dimensional time-dependent detached eddy simulation (DES) turbulent model is used in the study. The free surface was simulated using flat-wave model linked with the volume of fluid method. The aim is to provide an enhanced analysis hitherto that is not possible by experimental approaches. The importance of this study emerges from our need to gain a better understanding of secondary currents, with an ultimate goal to permit better management of the dynamic flow features in a variety of engineering and/or environmental applications.

\section{MODEL SETUP AND BOUNDARY CONDITIONS}

A schematic diagram of the open channel, the computational domain details, and the relevant boundary conditions that have been employed in this investigation are shown in Fig. 1. The aspect ratio (AR), which is the main parameter in this study, is determined by the ratio of the horizontal width of the rectangular channel cross-section to the water layer depth. As shown in Fig. 1(a), the computational domain consists of two regions; the water (represented by red cut lines) and air (represented by blue dots). The height $(H)$ and width $(B)$ of both water and air regions in the channel are the same for all cases used in the study. The channel streamwise length is kept constant for all cases, i.e. $L=500 \mathrm{~mm}$. The water layer depth $H=30 \mathrm{~mm}$, and the two channel widths $B=30 \mathrm{~mm}$ and $360 \mathrm{~mm}$, are used to produce the channel aspect ratios $\mathrm{AR}=1$ and 12 , respectively. The inlet velocity at the channel inlet boundary is uniform for all cases in this study with a magnitude equal to $0.75 \mathrm{~m} / \mathrm{s}$. All wall boundaries in contact with 
(a)

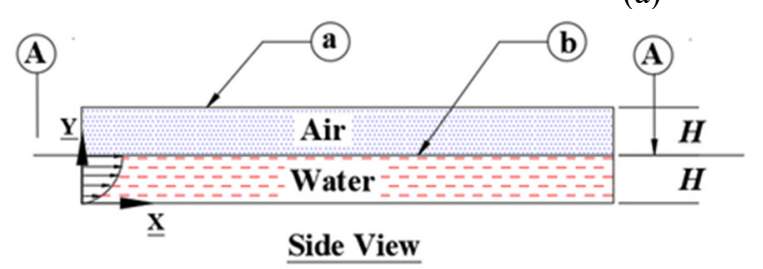

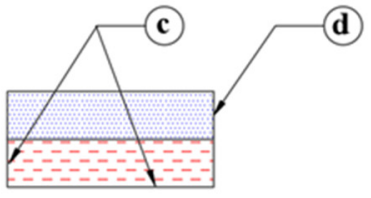

Front View

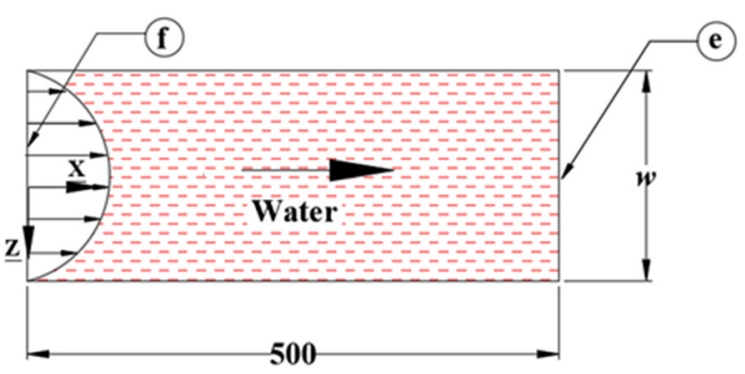

Section A-A

(a) Pressure outlet; (b) Water-air interface; (c) No-slip wall (water); (d) Slip wall (air); (e) Outlet boundary; and (f) Velocity inlet.

(b)

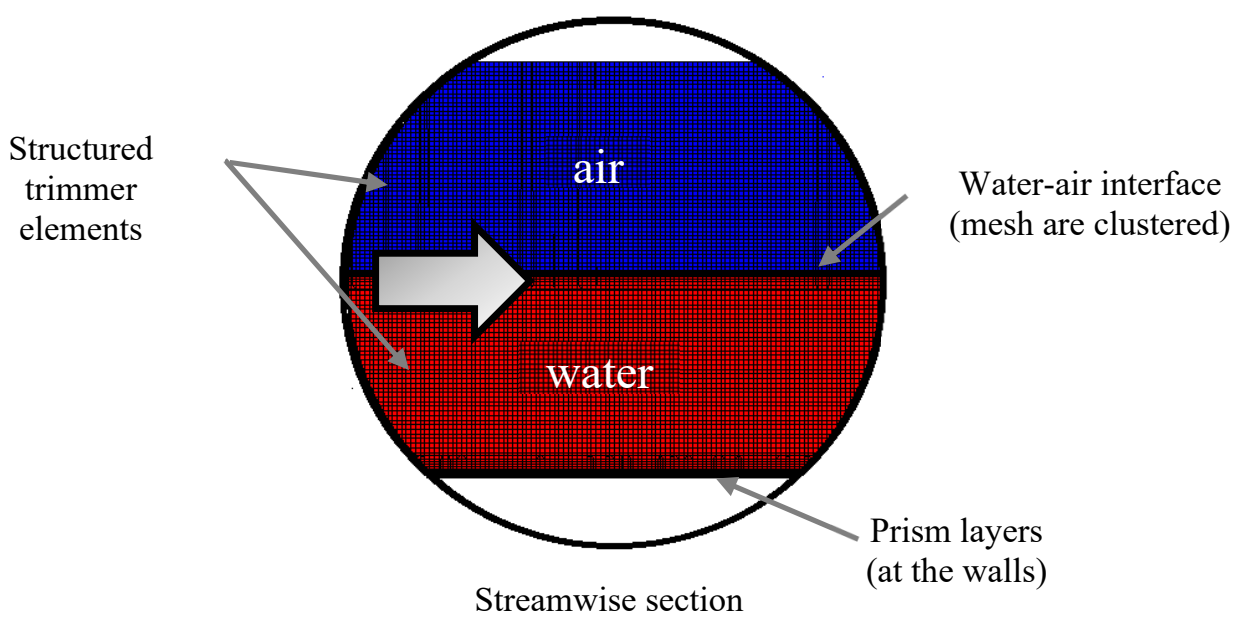

Figure 1: (a) A computational model with appropriate boundary conditions; and (b) Section through the computational domain in the streamwise direction [8].

the water region are considered as a no-slip smooth boundary, while all sidewalls that are in contact with the air region are considered as a slip boundary. The top surface of the air (labelled with letter "a" in Fig. 1) is treated as a pressure outlet boundary. The hydrostatic pressure of the flat wave model [7] is used as a boundary for the pressure, and the outflow boundary is used for the velocity in the water region at the outlet (labelled with letter "e" in Fig. 1). To reduce the effect of the numerical reflection of the waves from the boundaries, a 
damping factor is used for this purpose. The fully-developed turbulent velocity profile, which is used as an inlet boundary condition (labelled with letter "f" in Fig. 1), is obtained by periodically mapping the velocity components and turbulent fluctuations distribution from the $y$-z plane (see Fig. 1) located mid-way $(x / L=0.5)$ in the streamwise direction $x$, until the mean velocity profiles become almost identical across the channel flow at different streamwise locations [8]. The reason for extracting the data at this streamwise location rather than at the outlet boundary is to avoid the influences of the exit boundary condition since the velocity is observed to increase as it approaches the channel exit due to a slight decline in the water level.

\section{COMPUTATIONAL METHODOLOGY}

The open channel flow is simulated using Siemens PLM's STAR-CCM+ [9] with a structured trimmer mesh. The trimmer meshing model utilizes a template mesh that is constructed from hexahedral cells from which it cuts or trims the core mesh using the structured hexahedral surface mesh as the starting input [9]. First-order implicit marching in time and second-order differencing in space are used to discretize the governing equations. The time-dependent governing equations comprise a continuity equation for conservation of mass, three conservation of momentum equations, and conservation of energy equation. Each of these equations can be expressed in a general form by the transport of a specific scalar quantity $\phi$ per unit mass, represented in a continuous integral form as [10]:

$$
\frac{\partial}{\partial t} \int_{\mathrm{CV}} \rho \phi d \mathrm{~V}+\oint_{A} \mathbf{n} \cdot(\rho \phi \mathbf{u}) d A=\oint_{A} \mathbf{n} \cdot\left(\Gamma_{\phi} \boldsymbol{\nabla} \phi\right) d A+\int_{\mathrm{CV}} S_{\phi} d \mathrm{~V}
$$

where CV in eqn (1) represents the three-dimensional control volume over which the volume integration is carried out, $A$ is the bounding surface of the control volume. The terms in eqn (1) from left to right are the rate of change of the total quantity of the fluid property $\phi$ in the control volume, the rate of change of the property $\phi$ due to the convection flux across the bounding surface of the control volume, the rate of change of the property $\phi$ due to the diffusive flux across the bounding surface of the control volume, and the volumetric source in the control volume. The unit vector $\mathbf{n}$ in eqn (1) is the outward normal vector to the surface, $\mathbf{u}$ is the instantaneous velocity vector, $\rho$ is the density, and $\Gamma_{\phi}$ is the diffusion coefficient.

Improved delayed detached-eddy simulation (IDDES) model [11]-[13] is a modified turbulent model that employs Reynolds-Averaged Navier-Stokes (RANS) equation at the near-wall regions and Large-eddy simulation (LES) at the rest of the flow. The model was originally formulated by replacing the distance function in the Spalart-Allmaras model with a modified distance function. The $k-\varepsilon$ Shear Stress Transport (SST) turbulence model is a two-equation eddy-viscosity model [14], [15] and has been selected as the RANS part of the DES turbulence model in this study. The $k-\varepsilon$ SST model solves additional transport equations for turbulent kinetic energy $k$ and specific dissipation rate $\varepsilon$, from which the turbulent kinematic viscosity $\left(v_{\mathrm{t}}=k / \varepsilon\right)$ can be derived. The transport equations of $k$ and $\varepsilon$ are described in [15].

Since the flow field under investigation involves two immiscible fluids, a numerical model to handle two-phase flow is required. The volume of fluid (VOF) model [16] is a simplified multiphase approach that is well suited to simulate flows of several immiscible fluids and is capable of resolving the interface between the mixture phases. In a VOF simulation, the basic model assumption is that all phases share the same velocity and pressure and no additional modelling of inter-phase interaction is required. The normalized variable diagram provides the methodology used in constructing high-resolution schemes [17]. The 
Compressive Interface Capturing Scheme for Arbitrary Meshes (CICSAM) [18] and the High-Resolution Interface Capturing Scheme (HRIC) [19] are the most commonly used highresolution schemes for interface capturing with the VOF model. The HRIC scheme is used to capture the interface in the present work. In high-resolution schemes, an additional condition is required, the convective boundedness criterion must be satisfied along with the local Courant-Friedrichs-Lewy (CFL) condition. The CFL condition is a necessary condition for numerical stability. If an explicit time marching solver is used, then a Courant number less than one is typically required. Implicit solvers, like the one used in this study, are less sensitive to numerical instability and larger values of Courant number may be tolerated [9].

\section{MESH GENERATION}

In the present study, structured-trimmer elements are used to mesh the computational domain. Preliminary open-channel flow simulations utilizing the flow field that resembles experimental studies, which are carried out in a recirculating open channel flume at the Hydraulic Engineering Research Laboratory at the University of Windsor [20], were performed first for cell sensitivity inspection and model validation purposes. Detailed validations were carried out by comparing the streamwise velocity and root-mean-square of turbulence level profiles from experimental and computational results. In each case, flow field parameters were checked and compared with experimental results. Furthermore, successive grids were compared to determine whether or not there was a change in the mean characteristics. The results for the mean flow quantities and cell independence study were very similar to those reported earlier in [8], [21], [22] and are not repeated here for brevity. In this study, the total number of cells used for the computation is 1.3 and 8.7 million elements for $\mathrm{AR}=1$ and 12 , respectively. In the present study, ten layers of fine prism cells, packed within $1.5 \mathrm{~mm}$ using a stretching factor of 1.5 , are employed to resolve the wall effect. The non-dimensional wall-normal distance $\left(y^{+}\right)$value is less than two everywhere in the entire computational domain, which lies in the viscous sub-layer. The cells are also clustered along the water-air interface to reduce the numerical diffusion and preserve the sharpness of the water-air interface. To select the proper time step $(\Delta t)$, different time steps were tried to satisfy the local Courant-Friedrichs-Lewy (CFL) condition [9]. The final time step was set as $2 \times 10^{-4} \mathrm{~s}$, yielding a Courant number less than 0.5 in the entire computational domain. Five internal iterations were employed at each time step. The physical time that is used for averaging the transient quantities is $15 \mathrm{~s}$ for all cases of this investigation. This time is initiated after the instantaneous flow field reaches its steady condition. The numerical results are considered to have converged when the scaled continuity and momentum residuals fall below $10^{-6}$.

\section{RESULTS}

In this section, vector plots and/or color contours of the mean velocity field of an open channel flow are presented and discussed. Two cases are discussed in this paper to highlight the effect of aspect ratio (AR) on the formation of secondary currents; one represents a small aspect ratio $(\mathrm{AR}=1)$ and the other one represents a much larger aspect ratio $(\mathrm{AR}=12)$ which is used as a reference case. In the following results, $x$-, $y$-, and $z$-axes represent streamwise, vertical, and horizontal locations respectively; while $U, V$, and $W$ represent the corresponding mean velocity components. 


\subsection{Boundary layer characteristics}

The purpose of this section is to provide an extra validation of the current computational results of the open channel flow, and also to document the characteristics of the boundary layer flow. To confirm that the current results conform to the published results, a comparison between the streamwise velocity $U$ profile in the fully-developed region for the $\mathrm{AR}=12$ case and well-documented channel flow results is made. The bulk velocity $U_{\mathrm{b}}$ of the channel flow in this study is $0.75 \mathrm{~m} / \mathrm{s}$. The current results displayed that the velocity profile of the channel flow varies throughout the water layer depth $H$, which suggests that the boundary layer can be assumed to extend to the free surface [23], [24]. However, the boundary layer thickness $\delta$ (perpendicular to the bed) in this study is estimated based on $0.995 U_{\mathrm{b}}$, and found to occupy $\sim 29.3 \%$ of the water layer depth $H$. Consequently, the displacement thickness $\delta^{*}$ and momentum thickness $\theta$ were estimated to be 0.58 and $0.41 \mathrm{~mm}$, respectively. The resulting shape factor is 1.41 and the Reynolds number based on the momentum thickness $\operatorname{Re}_{\theta}$ is $\sim 308$.

Fig. 2 shows distribution of the streamwise component of the mean velocity in the channel flow in terms of the dimensionless wall velocity $U^{+}\left(=u / u_{\tau}\right)$ and vertical axis $y^{+}\left(=y u_{\tau} / v\right)$. The friction velocity $u_{\tau}$, defined as $\vee_{\tau} / \rho$, was determined by the Clauser chart method [25], which is based on the assumption that the velocity profile follows a universal logarithmic form in the overlap region of the boundary layer. In this study, the friction velocity $u_{\tau}$ was estimated to be $39 \mathrm{~mm} / \mathrm{s}$. The corresponding skin friction coefficient $C_{f}$, defined by $2\left(u_{\tau} / U_{\mathrm{b}}\right)^{2}$, was found to be $5.41 \times 10^{-3}$. The viscous length scale $l_{v}$, defined by $\left(v / u_{\tau}\right.$, where $v$ is the kinematic viscosity of the water) was estimated to be $0.026 \mathrm{~mm}$. The present velocity profile was also compared with the experimental results of Balachandar and Bhuiyan [26] for smooth channel flow at a similar Reynolds number. The present results are in good agreement with the previous channel flow data.

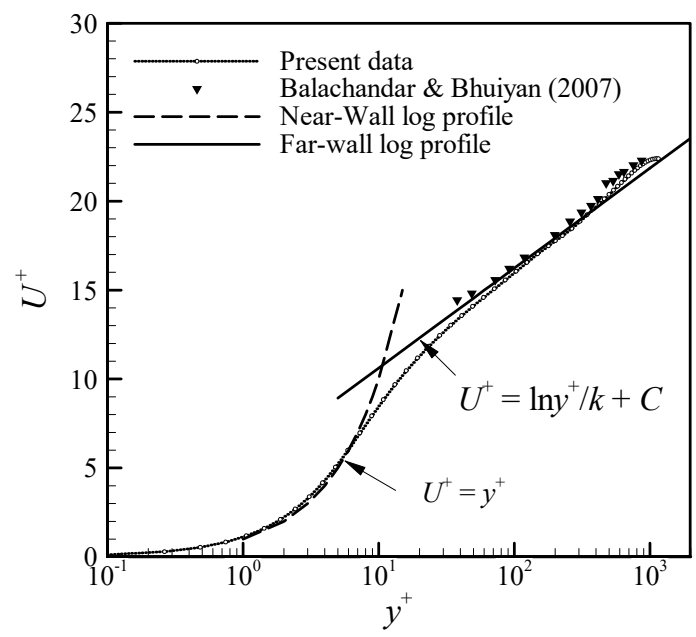

Figure 2: Mean streamwise velocity distribution of the smooth open-channel flow using the inner coordinate. The current velocity data for $\mathrm{AR}=12$ is compared with experimental results. 


\subsection{Mean velocity field}

Fig. 3 shows two vector plots of the mean velocity field in $y$-z plane for aspect ratios AR $=1$ and 12 , respectively, extracted from the fully-developed flow region of an open channel flow. In these plots, the colour contour, which represents the mean streamwise velocity component, is also shown in the plots to provide a better description of $3 \mathrm{D}$ velocity field. In this figure, locations are normalized by the water layer depth $H$ and velocities are normalized by the bulk velocity $U_{\mathrm{b}}$. Note that only some vectors are shown to avoid cluttering on the figures. The vector plot shown in Fig. 3(a) clearly illustrates the formation a pair of strong counter-rotating recirculation zones near the bottom corners of the channel for the $\mathrm{AR}=1$ case, whose axes are aligned with the streamwise direction $x$; one of the pair resides near the bottom bed while other one is relatively bigger and resides near the sidewalls. Furthermore, there is a strong downward flow in the mid-vertical plane driven by the recirculation zones that reside near the sidewalls which meets with a relatively weaker upward flow driven by the lower recirculation zones. Fig. 3(b) shows that the behavior of the AR $=12$ case is very different. It shows the formation of a pair of counter-rotating recirculation zones in the bottom corners of the channel. However, the strengths of the recirculation zones near the bed are quite energetic and they appear to span almost the whole flow depth, while the zones that reside near the sidewalls appear weak and confined in a narrow region near the sidewalls (see the region highlighted by a red square). In the core region of this wide channel (AR $=12)$, Fig. 3(b) shows that the magnitude of the mean vertical $V$ and horizontal $W$ velocity components
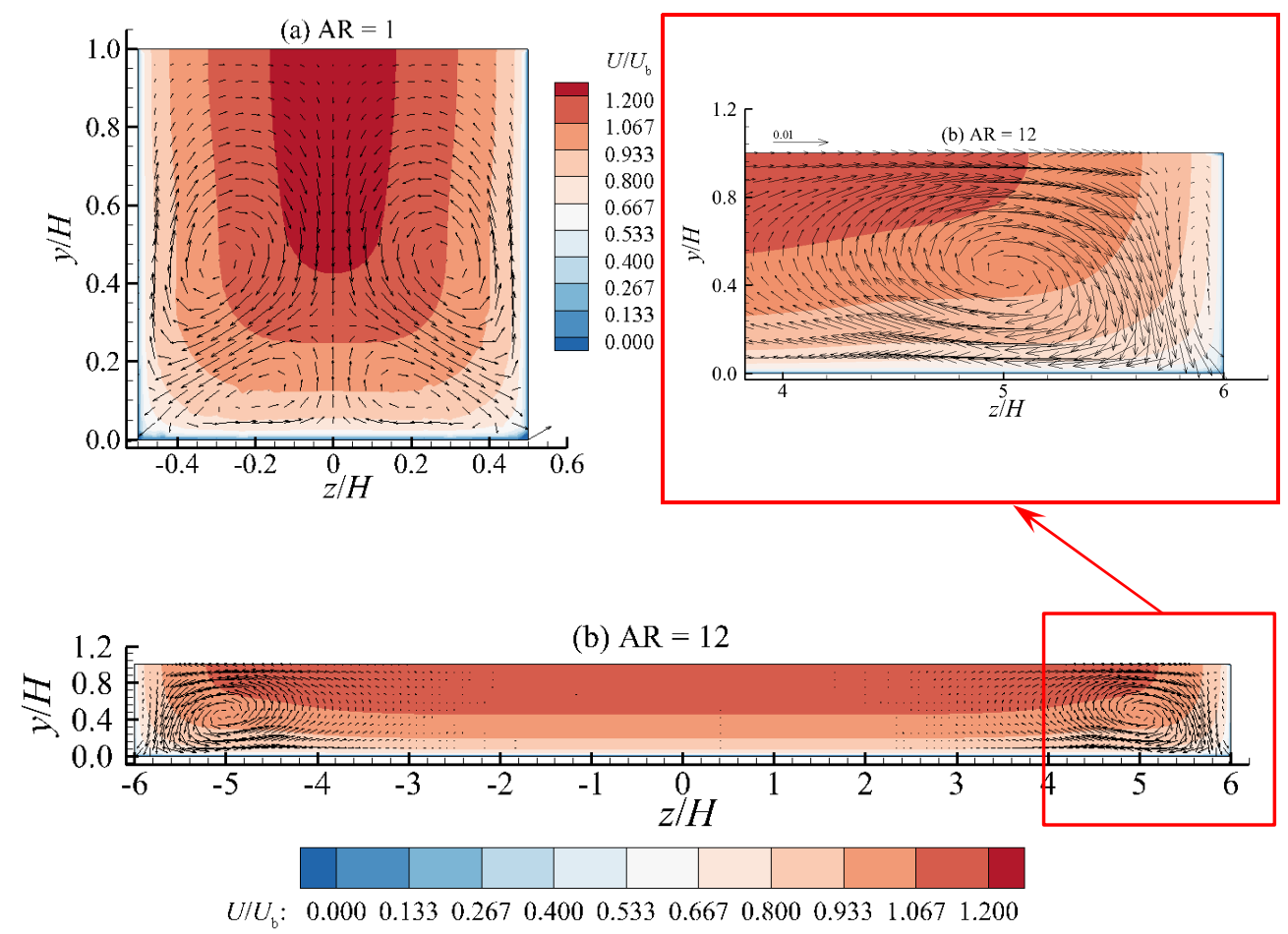

Figure 3: Mean velocity field for an open channel flow in the fully-developed flow region for (a) $\mathrm{AR}=1$; and (b) $\mathrm{AR}=12$. The colour contour represents the normalized mean streamwise velocity $U / U_{\mathrm{b}}$ component. 
are almost zero. On the other hand, the colour contour generally illustrates that the mean streamwise velocity is zero at the solid walls and increase gradually to reach its maximum magnitude in the core region of the channel flow for $\mathrm{AR}=1$ and 12 as shown in Figs 3(a) and 3(b). However, the darker brown colour in the core region in Fig. 3(a) compared to Fig. 3 (b) indicates that the magnitude of $U$ for $\mathrm{AR}=1$ is larger than the $\mathrm{AR}=12$ case. This may be attributed to formation of the boundary layers on the solid bottom and side walls, which displaces the flow vertically and horizontally, respectively, and thus enhance $U$.

To quantify the effect of the AR on the velocity field, the mean streamwise $U$ and vertical $V$ velocity profiles along the vertical mid-plane $(z / H=0)$ of the flow depth are presented in Fig. 4. In this figure, $U$ and $V$ are normalized by the bulk velocity $U_{\mathrm{b}}$ and the vertical locations $y$ by the flow depth $H$. As shown in Fig. 4(a), $U / U_{\mathrm{b}}$ for the AR $=1$ case appears larger than the $\mathrm{AR}=12$ case. The maximum magnitude of $U / U_{\mathrm{b}}$ for the $\mathrm{AR}=1$ and 12 is $\sim 1.24$ and $\sim 1.16$, respectively. Moreover, Fig. 4(b) illustrates a relatively strong downward flow (negative $V / U_{\mathrm{b}}$ ) from the free surface towards the bed, and an upward flow (positive $V / U_{\mathrm{b}}$ ) originated near the bed for $\mathrm{AR}=1$. The maximum magnitude of the downward and upward flows is $\left|V / U_{\mathrm{b}}\right| \approx 0.008$ and 0.005 , respectively. On the other hand, $V / U_{\mathrm{b}}$ is zero for the $\mathrm{AR}=$ 12 throughout the flow depth. For the mean horizontal velocity component $\left(W / U_{\mathrm{b}}\right)$, it is obviously zero at the vertical mid-plane for both cases because of the symmetry.

(a)

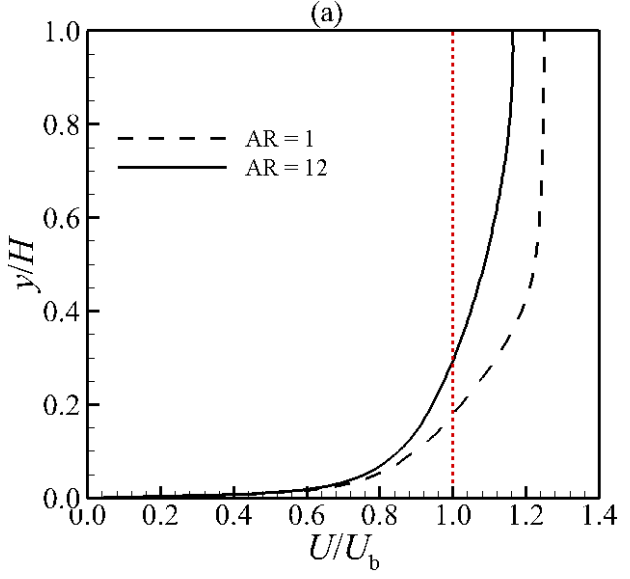

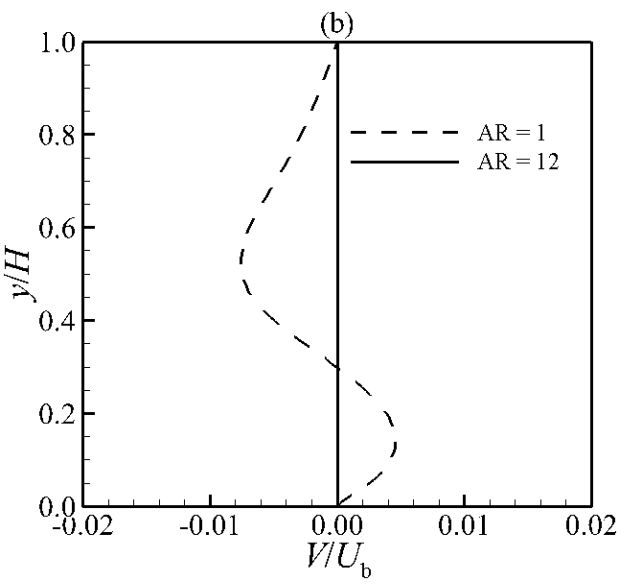

Figure 4: Variation of the mean velocity profiles of channel flow in the fully-developed region with the flow depth $y / H$ extracted from the mid-vertical plane $(z / H=0)$ for $\mathrm{AR}=1$ and 12. (a) Streamwise $U$ profiles; and (b) Vertical $V$ velocity profiles.

\subsection{Mean turbulent kinetic energy}

To highlight the effect of the aspect ratio on the energy distribution in the flow, Fig. 5 displays the turbulent kinetic energy $k$ as a color contour superimposed on the mean velocity field in $y-z$ plane. In this figure, $k$ is normalized by $U_{\mathrm{b}}{ }^{2}$. Fig. 5(a) and (b) illustrate that the maximum turbulent kinetic energy $k$ occurs in the layer adjacent to the bottom and side walls (brown color), while the minimum $k$ occurs in the core region of the flow far from the solid walls (blue color). To have a better perception of the effect of the AR, $k$ profiles are shown in Fig. 6 at horizontal locations $z / B=0,0.25$, and 0.42 . Here $B$ represents the width of the channel. 
Note that $z / B=0$ represents the vertical mid-plane and $z / B= \pm 0.5$ represents the location of the sidewalls. Consistent with Figs 5 and 6(a)-(c) show that the peak value of $k$ is located near the bottom solid wall at $y / H \approx 0.016$ with a magnitude of $k / U_{\mathrm{b}}{ }^{2} \approx 0.014 \pm 0.001$ for all $z / B$ locations. Far from the bed, the magnitude of $k$ is generally smaller over most of the flow depth for the $\mathrm{AR}=1$ case compared to the $\mathrm{AR}=12$ case at $z / B=0$. However, the magnitude of $k$ appears to slightly increase for the AR $=12$ near the free surface as it approaches the sidewalls, but the rate of increase of $\mathrm{k}$ for the $\mathrm{AR}=1$ is much larger until it becomes almost uniform at $z / B=0.42$ with a magnitude of $k / U_{\mathrm{b}}^{2} \approx 0.01$.
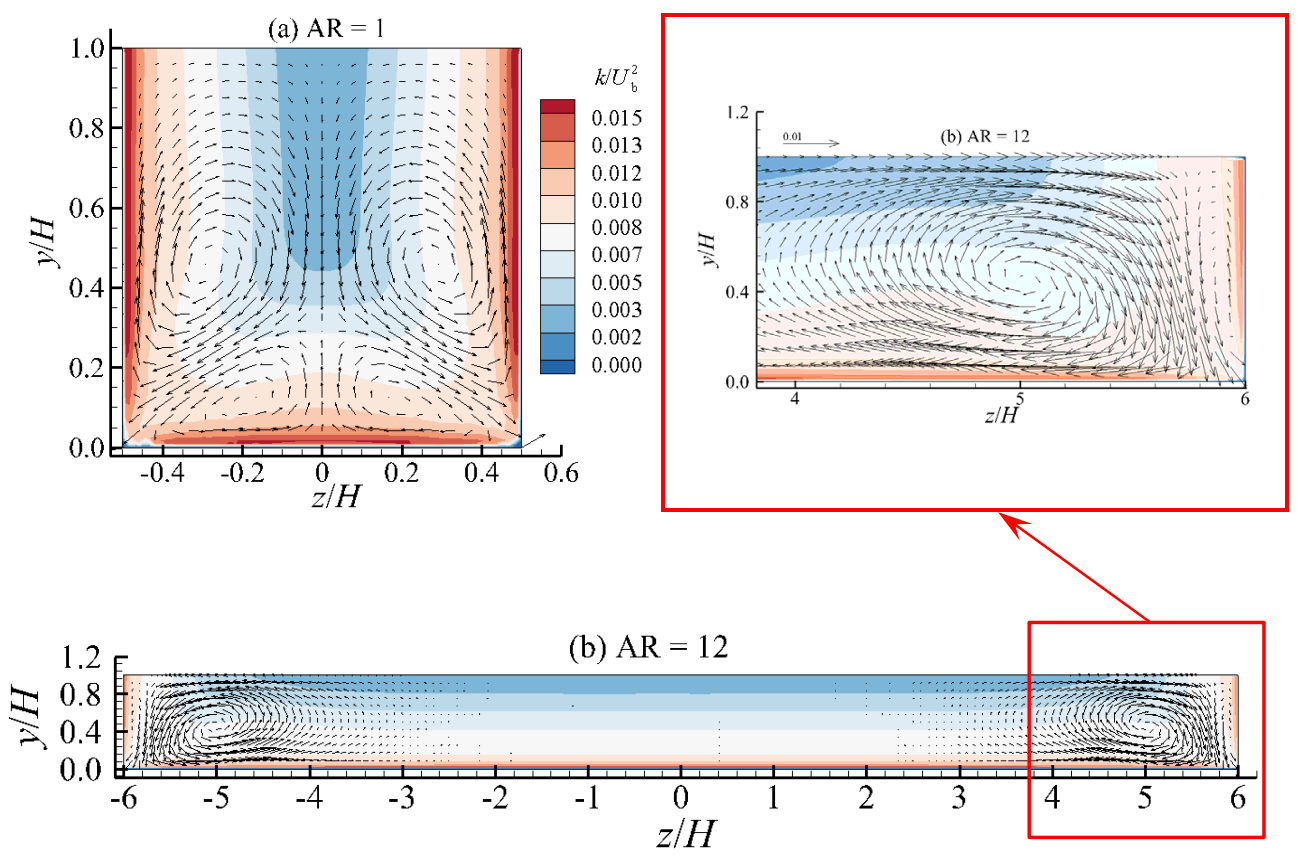

Figure 5: Mean velocity field for an open channel flow in the fully-developed flow region for (a) $\mathrm{AR}=1$; and (b) $\mathrm{AR}=12$. The colour contour represents the normalized turbulent kinetic energy $k / U_{\mathrm{b}}^{2}$.
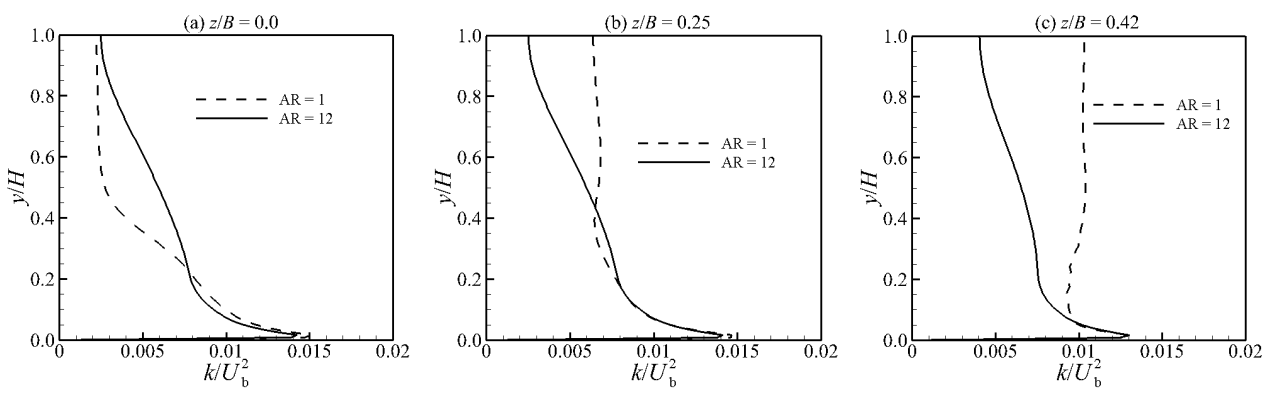

Figure 6: Variation of the normalized turbulent kinetic energy $k / U_{\mathrm{b}}^{2}$ profiles of channel flow in the fully-developed region with the flow depth $y / H$ for $\mathrm{AR}=1$ and 12 extracted at: (a) $z / B=0$; (b) $z / B=0.25$; and (c) $z / B=0.42$. 


\section{CONCLUSIONS}

From the above, the following conclusions can be drawn:

- A pair of counter-rotating mean recirculation zones is formed at the bottom edges of a straight open channel flow; one near the sidewall and one near the bed. The recirculation zone that reside near the bed is dominant and energetic and almost spans the entire flow depth for $\mathrm{AR}=12$, while the one that reside near the sidewall appears weaker, more confined, and its size is much smaller. Contrary to this behavior, the near-bed recirculation zone becomes more confined and relatively smaller for the AR $=1$ case compared to the $\mathrm{AR}=12$ case, while the ones that reside near the sidewalls become much more energetic and larger in size, and their influence extend up to the free surface.

- The distribution of the turbulent kinetic energy $k$ indicates that the maximum value occurs in the layer adjacent to the solid walls for both aspect ratios, and decreases gradually away from the solid walls towards the core region towards the free surface. However, the magnitude of $k$ appears to increase with a much higher rate as the sidewalls are approached for $\mathrm{AR}=1$ compared to the $\mathrm{AR}=12$ case, at corresponding $z / B$. It can be inferred that the decrease of the AR causes an increase of the components of turbulent velocity fluctuations.

\section{ACKNOWLEDGEMENTS}

This research was made possible by the facilities of the Shared Hierarchical Academic Computing Network (SHARCNET: www.sharcnet.ca) and Compute/Calcul Canada.

\section{REFERENCES}

[1] Bradshaw, P., Turbulent secondary flows. Annual Review of Fluid Mechanics, 19(1), pp. 53-74, 1987.

[2] Einstein, H. \& Li, H., Secondary currents in straight channels. EOS, Transactions American Geophysical Union, 39(6), pp. 1085-1088, 1958.

[3] Nezu, I. \& Rodi, W., Experimental study on secondary currents in open-channel flows. Proceedings of 21st IAHR Congress, Melbourne, pp. 115-119, 1985.

[4] Nezu, I., Nakagawa, H. \& Tominaga, A., Secondary currents in a straight channel flow and the relation to its aspect ratio. In Turbulent Shear Flows, 4, pp. 246-260, 1985.

[5] Nezu, I. \& Nakagawa, H., Turbulence in Open Channel Flows, Balkema: Netherlands, 1993.

[6] Tominaga, A., Nezu, I., Ezaki, K. \& Nakagawa, H., Three-dimensional turbulent structure in straight open channel flows. Journal of Hydraulic Research, 27(1), pp. 149-173, 1989.

[7] Fenton, J.D., The Cnoidal theory of water waves. Developments in Offshore Engineering, pp. 55-100, 1999.

[8] Nasif, G., Balachandar, R. \& Barron, R.M., Supercritical flow characteristics in smooth open channels with different aspect ratios. Physics of Fluids, 32(10), p. 105102, 2020.

[9] Siemens PLM, Global, STAR-CCM+13.06.012: User Manual, 2018.

[10] Malalasekera, W. \& Versteeg, H.K., An Introduction to Computational Fluid Dynamics: The Finite Volume Method, 2nd edn, Pearson Education Ltd.: Harlow, UK, 2007.

[11] Menter, F.R. \& Kuntz, M., Adaptation of eddy-viscosity turbulence models to unsteady separated flow behind vehicles. The Aerodynamics of Heavy Vehicles: Trucks, Buses, and Trains, Springer: Berlin, Heidelberg, pp. 339-352, 2004. 
[12] Travin, A., Shur, M., Strelets, M.M. \& Spalart, P.R., Physical and numerical upgrades in the detached-eddy simulation of complex turbulent flows. Advances in LES of Complex Flows, pp. 239-254, 2002.

[13] Spalart, P.R., Deck, S., Shur, M.L., Squires, K.D., Strelets, M.K. \& Travin, A., A new version of detached-eddy simulation, resistant to ambiguous grid densities. Theoretical and Computational Fluid Dynamics, 20(3), p. 181, 2006.

[14] Wilcox, D.C., Simulation of transition with a two-equation turbulence model. AIAA Journal, 32(2), pp. 247-255, 1994.

[15] Wilcox, D.C., Turbulence Modelling for CFD, vol. 2, DCW Industries: La Canada, CA, pp. 103-217, 1998.

[16] Hirt, C.W. \& Nichols, B.D., Volume of fluid (VOF) method for the dynamics of free boundaries. Journal of Computational Physics, 39(1), pp. 201-225, 1981.

[17] Leonard, B.P., The ULTIMATE conservative difference scheme applied to unsteady one-dimensional advection. Computer Methods in Applied Mechanics and Engineering, 88(1), pp. 17-74, 1991.

[18] Ubbink, O. \& Issa, R.I., Method for capturing sharp fluid interfaces on arbitrary meshes. Journal of Computational Physics, 153, pp. 26-50, 1999.

[19] Muzaferija, S., A two-fluid Navier-Stokes solver to simulate water entry. Proceedings of 22nd Symposium on Naval Architecture, pp. 638-651, 1999.

[20] Heidari, M., Balachandar, R., Roussinova, V. \& Barron, R.M., Characteristics of flow past a slender, emergent cylinder in shallow open channels. Physics of Fluids, 29(6), p. $065111,2017$.

[21] Nasif, G., Balachandar, R. \& Barron, R.M., Influence of bed proximity on the threedimensional characteristics of the wake of a sharp-edged bluff body. Physics of Fluids, 31(2), p. 025116, 2019.

[22] Nasif, G., Balachandar, R. \& Barron, R.M., Effect of gap on the flow characteristics in the wake of a bluff body near a wall. International Journal of Computational Methods and Experimental Measurements, 7(4), pp. 305-315, 2019.

[23] Kirgoz, M.S. \& Ardiclioglu, M., Velocity profiles of developing and developed open channel flow. Journal of Hydraulic Engineering, 123(12), pp. 1099-1105, 1997.

[24] Auel, C., Albayrak, I. \& Boes, M., Turbulence characteristics in supercritical open channel flows: Effects of Froude number and aspect ratio. Journal of Hydraulic Engineering, 140(4), p. 04014004-1-15, 2014.

[25] Clauser, F., Turbulent boundary layers in adverse pressure gradient. Journal of the Aerospace Sciences, 21, pp. 91-108, 1954.

[26] Balachandar, R. \& Bhuiyan, F., Higher-order moments of velocity fluctuations in an open channel flow with large bottom roughness. Journal of Hydraulic Engineering, 133, pp. 77-87, 2007. 